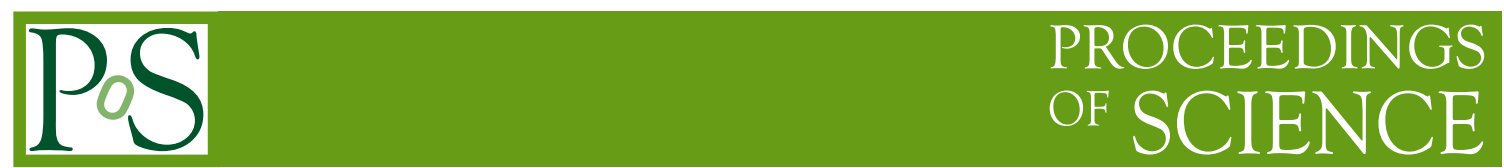

\title{
Search for new resonances in high-mass diphoton final states using proton-proton collision data collected with the ATLAS detector
}

\author{
Yufeng Wang, on behalf of the ATLAS Collaboration ${ }^{a, b, *}$ \\ ${ }^{a}$ Laboratoire de physique nucléaire et de hautes énergies, \\ 4 Place Jussieu, Tour 22, ler étage, Paris, France \\ ${ }^{b}$ University of Science and Technology of China, \\ 96 JinZhai Road, Baohe District, Hefei, Anhui, P.R.China \\ E-mail: yufeng.wang@cern.ch
}

\begin{abstract}
A search for new resonances decaying to two photons is performed, using $139 \mathrm{fb}^{-1}$ of $p p$ collision data at $\sqrt{s}=13 \mathrm{TeV}$ collected by the ATLAS detector at the LHC. A spin-0 signal predicted by theories with extended Higgs sector and a spin-2 graviton excitation using a warped extradimension benchmark model are searched for. No significant deviation from the Standard Model is observed. Upper limits are set on the production cross-section times branching ratio as a function of the resonance mass and width.
\end{abstract}

40th International Conference on High Energy physics - ICHEP2020

July 28 - August 6, 2020

Prague, Czech Republic (virtual meeting)

${ }^{*}$ Speaker 


\section{Introduction}

The presented search for a new high-mass resonance decaying to two photons is carried out using $139 \mathrm{fb}^{-1}$ of $p p$ collision data recorded by the ATLAS detector[1] at the LHC. Two kinds of signals are searched for: a spin- 0 resonant state $(X)$ predicted by many models with Higgs sector extensions, and a spin-2 graviton $\left(G^{*}\right)$ which is the lightest Kaluza-Klein (KK) excitation in the Randall-Sundrum model[2] with one extra dimension (RS1). In order to determine the signal yield, analytical functions describing the signal and background shapes are used to fit the diphoton invariant mass spectrum. In the absence of significant signal excesses, upper limits are placed on the fiducial (total) production cross-section times branching ratio as a function of resonance mass and width (coupling) for the spin-0 (spin-2) signal.

\section{Analysis overview}

This analysis is performed with data collected by the ATLAS detector from 2015 to 2018 using diphoton trigger. The analysis selection is optimized and unified for both spin- 0 and spin- 2 searches. Events with at least two photon candidates and $m_{\gamma \gamma}>150 \mathrm{GeV}$ are selected. The photon candidates are required to fulfill the tight photon identification and isolation criteria[3]. Kinematic cuts set on photon transverse energy $p_{T}$ relative to diphoton invariant mass $m_{\gamma \gamma}$ are also applied to further reduce the background.

Parametric models are used to describe the signals with different resonance mass and width. For signals with narrow decay width, their shape is dominated by the detector resolution and modeled by a double-sided Crystal Ball function. The true lineshape of large-width signals is obtained by convolving the resolution function and the lineshape at generator level.

The procedure of the background estimation is common for spin- 0 and spin- 2 searches. Two main continuum background components are considered: real $\gamma \gamma$ events ("irreducible background") modeled from MC simulation, and $\gamma j, j j$ events ("reducible background") with jets misidentified as photons, modeled from data control regions where the photon identification requirement is inverted. The two components are normalized according to their respective fractions measured in data. The analytical function describing the continuum background is determined and validated by fitting the total background template using the signal-plus-background model. The functional form is chosen to be flexible enough to capture any potential variations of the background template. Figure 1 shows the diphoton invariant mass distribution of the events passing the selection along with the background-only fit. The dominant systematic uncertainties of this analysis come from the photon energy resolution and the choice of background function.

\section{Results}

Results of this analysis are presented as a function of both resonance mass and width (coupling). A local $p$-value $\left(p_{0}\right)$ for the background-only hypothesis is calculated using the asymptotic approximation. No significant excess from the Standard Model expectation is observed. The largest deviation is found at $m_{X}=684 \mathrm{GeV}$ for narrow width spin- 0 and spin- $2\left(k / \bar{M}_{p l}=0.01\right)$ signals. A $3.29 \sigma$ local significance is computed, corresponding to about $1.3 \sigma$ global significance considering 


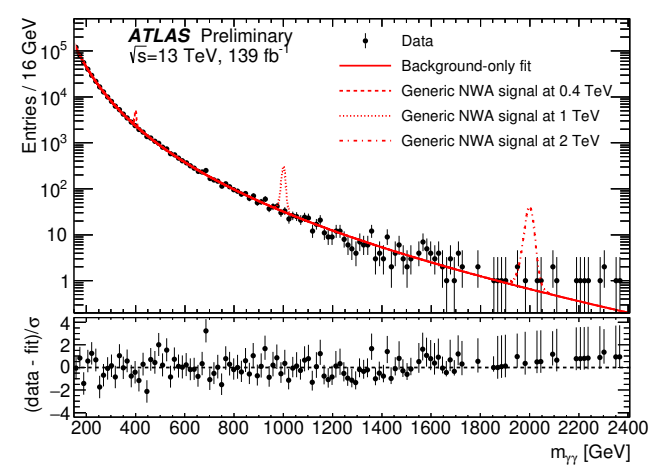

Figure 1: Background-only fit to data (black dots) as a function of the diphoton invariant mass $m_{\gamma \gamma}$ with several generic narrow-width signal shapes overlaid (dotted lines).[4]

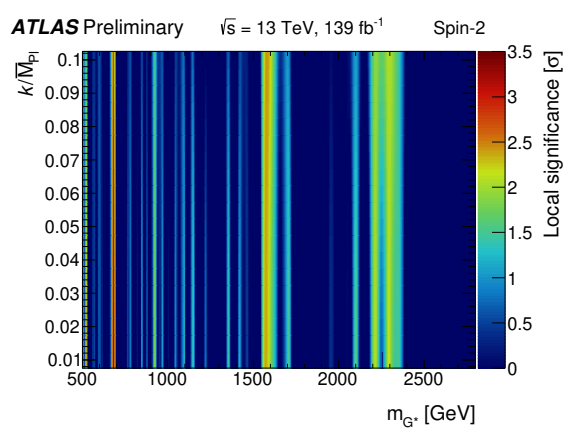

(a)

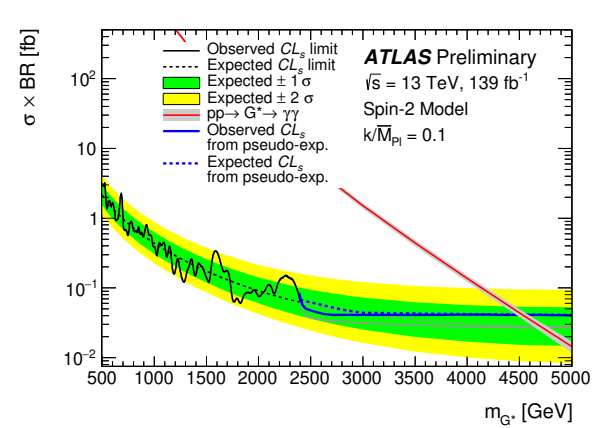

(b)

Figure 2: (a) Local $p_{0}$ with the background-only hypothesis and (b) total production cross-section times branching ratio to two photons of the lightest KK graviton as a function of $m_{G^{*}}$ for $k / \bar{M}_{p l}=0.1$. Crosssection predictions for the RS1 model are also shown in the figure.[4]

look-elsewhere effect. The expected and observed 95\% confidence level exclusion limits on the cross-section times branching ratio to two photons are also computed. Results calculated from pseudo-experiments are provided in the region where only very few events in data are observed. As an example, spin-2 results are shown in Figure 2. More details can be found in Ref. [4].

\section{References}

[1] ATLAS Collaboration, The ATLAS Experiment at the CERN Large Hadron Collider, JINST 3 (2008) S08003.

[2] L. Randall and R. Sundrum, Large mass hierarchy from a small extra dimension, Phys. Rev. Lett. 83 (1999) 3370.

[3] ATLAS Collaboration, Electron and photon performance measurements with the ATLAS detector using the 2015-2017 LHC proton-proton collision data, JINST 14 (2019) P12006 [1908.00005].

[4] ATLAS Collaboration, Search for resonances decaying to photon pairs in $139 \mathrm{fb}^{-1}$ of $\mathrm{pp}$ collisions at $\sqrt{s}=13 \mathrm{TeV}$ with the ATLAS detector, ATLAS-CONF-2020-037, https://cds.cern.ch/record/2727744. 